\title{
La Calidad y Excelencia de la Membresía en la Organización
}

Dr. Rodrigo Flores-Guerrero Profesor de la Facultad de Ciencias Sociales, Universidad de Chile. Doctor en Psicología, Universidad de Barcelona (España); Magíster en Antropología y Desarrollo, Universidad de Chile.

(rflores@uchile.cl)

Palabras clave: Organización, Calidad, Recursos Humanos, Índice, Excelencia.

\section{Resumen}

La calidad se ha convertido en una verdadera necesidad estratégica en las organizaciones. En diversos lugares del mundo aparecen instituciones que pretenden desarrollar y promover actividades y acciones que incrementen la eficacia, la eficiencia y la consecución de la excelencia. Sin embargo, no disponemos, hasta el momento, de ninguna herramienta que nos entregue una medición fiable de cuál es la calidad de las personas en la organización. Este artículo presenta los resultados de un estudio que pretenden sentar las bases teóricas y metodológicas para la elaboración de una medida confiable, válida y científica de la contribución que realiza la membresía a la organización en la que trabaja.

\section{La calidad de la organización}

La calidad se ha convertido en una verdadera necesidad estratégica en las organizaciones. En diversos lugares del mundo aparecen instituciones que pretenden desarrollar y promover actividades y acciones que incrementen la eficacia, la eficiencia y la consecución de la excelencia. No obstante, la preocupación por la calidad no se debe al último producto instaurado por un momentáneo visionario. En Japón su introducción en los procesos de producción comenzó ya en los años cincuenta, bajo el influjo de Joseph Juran y de Edward Deming. A partir de estos primeros investigadores, poco a poco y laboriosamente, comienza a ganar terreno la preocupación por "hacer las cosas bien". Con cierto retraso, la influencia de la calidad se dejó sentir en EE.UU. en los años ochenta. El año 1992 en Europa se instauran los galardones y premios EFQM, haciéndose tan popular que hoy en día el movimiento hacia la calidad es un referente habitual.

El término calidad no se ha mantenido estático a lo largo del tiempo, por el contrario, ha ido evolucionando y modificando su orientación. En un primer momento introducir calidad en las organizaciones se asoció a un mayor coste: la inspección del producto y el rechazo de todo aquel que resultara defectuoso implicaba un primer coste, después se debía intentar recuperar de él todo lo que fuera posible, lo que llevaba a un segundo coste. Posteriormente se comienzan a aplicar técnicas estadísticas de muestreo para verificar y controlar los productos resultantes.

Un cambio importante en el concepto de calidad es pasar de la verificación controladora y de inspección de los productos en su elaboración, a intentar generarla desde sus orígenes, es decir, asegurar la calidad en el proceso de producción para evitar que éste genere productos defectuosos. Es así como en estos últimos años se habla de la gestión de la calidad total. La calidad total es un programa que se ha de desarrollar en todos los niveles de la organización, cuya finalidad es instaurar y preservar un ambiente en el que los empleados mejoren 
continuamente su capacidad para suministrar productos y servicios. En este sentido, persigue la satisfacción total de los clientes, de la propia organización y de la sociedad. El concepto de gestión aquí resulta clave, pues incluye diferentes aspectos como el aseguramiento, control, prevención, mejora, planificación y optimización de la calidad.

\section{La Calidad de los Recursos Humanos de la Organización}

Actualmente existe un gran interés, académico y profesional, por evaluar la actuación de las personas en sus lugares de trabajo. Ello, por cierto, no es ninguna novedad, desde hace mucho tiempo que las organizaciones han implementado por medio de sus departamentos de recursos humanos, medidas de evaluación del rendimiento de sus trabajadores. No obstante, pareciera que medir el desempeño laboral no bastara. Cada día son más las organizaciones que desean alcanzar un alto rendimiento y, en consecuencia, obtener mejores resultados. Pero, ¿qué significa exactamente obtener buenos resultados?. Por cierto, no se puede negar la importancia que tiene la rentabilidad, ella es un elemento básico que garantiza la viabilidad de la organización, al permitirle comprar insumos, pagar a contratistas, remunerar a los empleados, realizar inversiones, etc. Sin embargo, obtener una alta retribución económica es tan sólo una parte del proceso y no puede convertirse en el único patrón por el cual se deba valorar el éxito de una organización.

Habitualmente, los indicadores de más amplia difusión que se han utilizado para medir los efectos o resultados de los individuos en la organización toman en consideración valoraciones negativas, como si se tratara de controlar o reducir un mal con el que tenemos forzosamente que convivir. Este es el caso, por ejemplo, de las mediciones que se realizan sobre el absentismo, la accidentabilidad laboral o los índices de rotación del trabajador. Sin embargo, los patrones de valoración están cambiando. Desde la década de los años noventa, cuando comienzan a generalizarse los modelos de calidad y excelencia, las personas son entendidas como un factor clave en la consecución de los objetivos y los resultados que la organización se ha fijado (1)

Actualmente los cientistas sociales preocupados por el estudio de las organizaciones se encuentran utilizando expresiones del tipo "capital intelectual", "contribución a los resultados organizacionales", "activo humano" o bien "talento". Con todas ellas no pretenden otra cosa que poder describir una compleja dimensión, compuesta de numerosas facetas, y de una importancia y relevancia tan grande, que permita explicar en alguna medida el funcionamiento y el éxito de la organización.

La calidad de los recursos humanos es aquel potencial de contribución de los individuos a la consecución de los objetivos y resultados de la organización de la cual forman parte (FloresGuerrero, 2003). No cabe duda que lograr realizar la elaboración de este constructo y conseguir de él una medida confiable y válida, facilitaría y potenciaría la investigación científica en las organizaciones. Como sabemos, uno de los más importantes principios científicos - dentro del paradigma positivista - es el de la parsimonia. Lograr aglutinar en una sola variable la contribución que realiza el individuo en la organización en que trabaja se encuentra en estrecha relación con este postulado.

A fin de valorar adecuadamente este término, podemos pensar en una especie de continuo, donde en uno de sus extremos se encuentra la menor (inferior o baja) calidad de la membresía, mientras que en el otro extremo se encuentra una mayor (superior, alta) calidad de las personas. La calidad, en este sentido, incluye una serie de procesos culturales, sociales y psicológicos que tienen lugar en la organización. Bajo su alcance se puede aunar la percepción 
de los trabajadores sobre la cultura, los estilos de dirección y liderazgo, la percepción de la membresía de su nivel de desarrollo grupal, efectividad y rendimiento grupal, así como sus percepciones sobre la identificación y el compromiso, la motivación, la satisfacción, el estrés y el burnout.

La calidad de los recursos humanos $(\mathrm{CRH})$ es un término molar, que pretende describir una serie de comportamientos laborales que se encuentran estrechamente interrelacionados, pretendiendo aunar:

- Elementos básicos y fundantes de nuestras disciplinas. Encontramos en su interior dimensiones culturales, sociales y psicológicas. Este es el caso de términos como el de cultura, motivación, satisfacción, estrés, etc.

- Términos que obedecen a una variedad de teorías y modelos. Muchos de estos términos no han sido integrados bajo una sola teoría. Ello se debe, en parte, a la utilización de lo que Merton denominó "teorías de alcance medio", es decir, que explican fenómenos acotados, pero con gran intensidad.

- Términos que no presentan una articulación clara y sistemática. Ello ha impedido el desarrollo de estrategias y principios explicativos de carácter general e integradores.

En la figura nำ1 se presenta de forma esquemática el conjunto de dimensiones establecidas para la calidad de los recursos humanos de la organización. En él podemos observar:

- La presencia de tres niveles de análisis, individual, grupal y del sistema organizacional.

- Relaciones entre las dimensiones de calidad de los recursos humanos.

- La causalidad entre las dimensiones de la calidad se ha omitido expresamente. Ello se debe a que en muchas ocasiones se desconoce el comportamiento que adoptan cada una de las dimensiones en determinados contextos organizativos. Para dar un ejemplo, es clara la relación positiva entre satisfacción laboral y compromiso del trabajador, sin embargo algunos estudios (Bateman y Strasser, 1984) han indicado que el compromiso es un antecedente de la satisfacción, mientras que otros (Farkas y Tetrick, 1989) señalan que el orden causal entre estas dos dimensiones se invierte con el paso del tiempo, como si se tratara de efectos cíclicos o recíprocos. 
Figura 1

Modelo de calidad de los recursos humanos de la organización

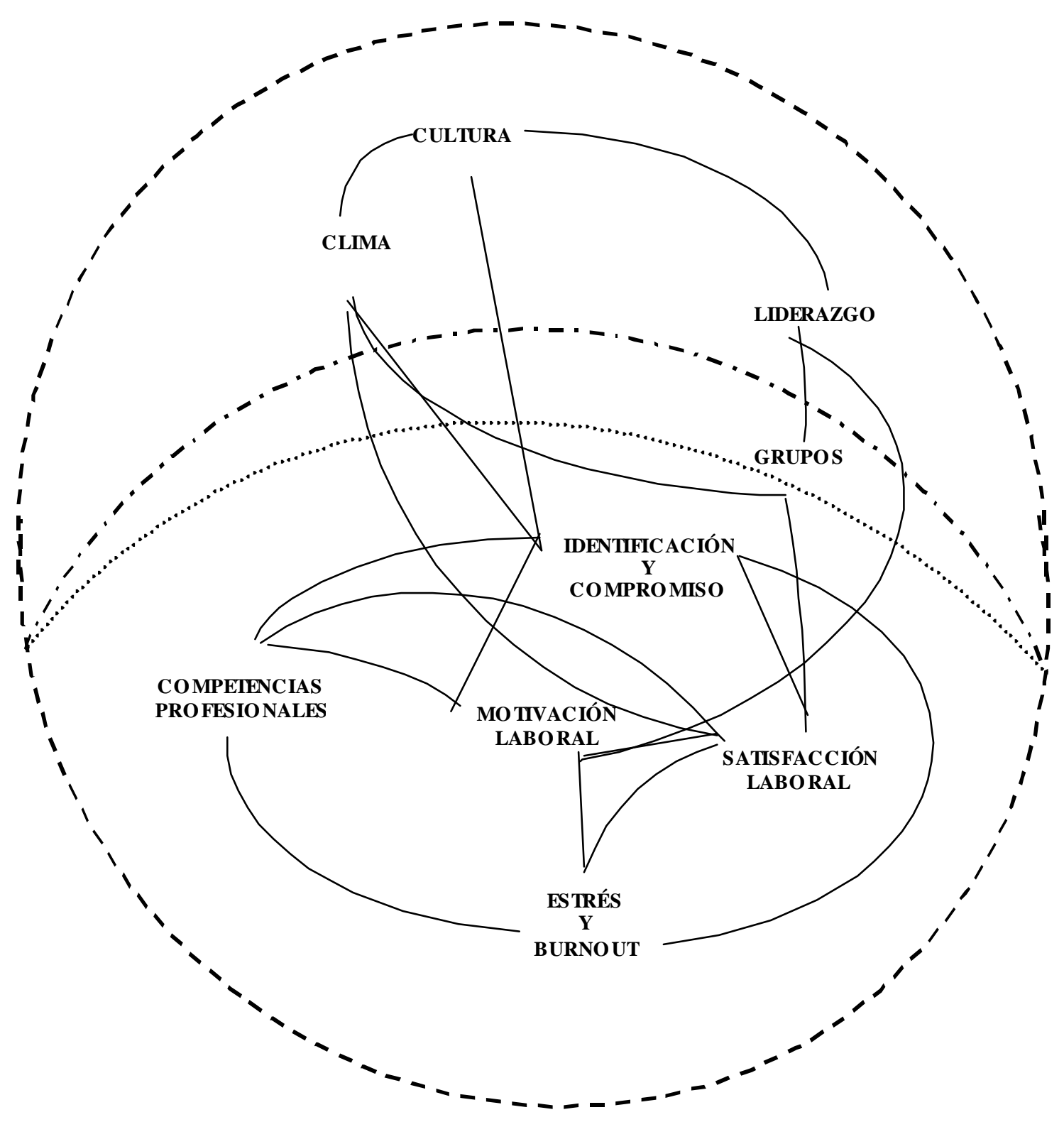


Algunas de las dimensiones que incluye el término calidad de los recursos humanos tienen efectos más directos e inmediatos sobre la ejecución del trabajo de la membresía. Este es el caso, por ejemplo, de a motivación, la identificación y el compromiso del individuo con la organización. Cuando se alcanzan altas cuotas en cada una de ellas, mejores resultados obtiene la membresía para la organización, mejor es el rendimiento de su trabajo y disminuye el absentismo laboral, la accidentalidad y propensión al abandono.

Otras dimensiones, en cambio, poseen efectos más indirectos en la efectividad organizativa, aunque su potencial contributivo a los resultados de la organización se mantiene intacto. Este es el caso, por ejemplo, de la cultura, el clima, los estilos de dirección y liderazgo, el desarrollo y la efectividad grupal, la satisfacción laboral, el estrés y el burnout. Si la membresía posee estados óptimos de satisfacción laboral no se garantiza con ello una mejora en la ejecución de los individuos; sin embargo, bajos niveles conducen a la frustración de las personas y a rendir menos. En otros casos, como el del estrés, sólo niveles óptimos producen una ejecución adecuada, pues niveles bajos de estrés conducen al inmovilismo del individuo (baja activación) mientras que elevados niveles conducen a la fatiga y la extenuación.

En este sentido, el presente artículo pretende responder las siguientes preguntas:

- ¿Las diferentes subdimensiones que han sido utilizadas tradicionalmente para evaluar el comportamiento humano en las organizaciones, pueden ser unificadas, teórica y empíricamente, en un solo constructo?

- En caso afirmativo, ¿Es posible la creación de un(os) índice(s) que operacionalice(n) este constructo?

- $Y$ en consecuencia, ¿Es posible realizar una adecuada descripción de sus principales componentes?

\section{Objetivos e Hipótesis del Estudio}

- Dar a conocer el constructo Calidad de los Recursos Humanos $(\mathrm{CRH})$ de la organización.

- Crear un(os) índice(s) capaz de operacionalizar la Calidad de los Recursos Humanos (CRH) de la organización

- Describir las subdimensiones que componen la Calidad de los Recursos Humanos (CRH) de la organización

Las hipótesis que guían nuestro estudio son las siguientes:

Hipótesis ${ }_{1}$.Si las diferentes dimensiones componentes de la $\mathrm{CRH}$ de la organización comparten una característica común definitoria de la $\mathrm{CRH}$, entonces cabe esperar que un índice de la $\mathrm{CRH}$ construido con ítems de esos sub-constructos permita obtener niveles elevados de confiabilidad.

Hipótesis 2. Si las diferentes dimensiones componentes de la $\mathrm{CRH}$ de la organización comparten una característica común definitoria de la $\mathrm{CRH}$, entonces cabe esperar que un índice de la $\mathrm{CRH}$ construido con sub-índices de esos sub-constructos permita obtener igualmente niveles elevados de confiabilidad.

Hipótesis 3. Si las diferentes dimensiones componentes de la $\mathrm{CRH}$ de la organización comparten una característica común definitoria de la $\mathrm{CRH}$, cabe esperar que una estimación de la $\mathrm{CRH}$ realizada con un número menor de ítems de los subconstructos (15-20), sea muy semejante a la obtenida con un número mayor de ellos (150-170). 
Hipótesis ${ }_{4}$. Igualmente, es presumible esperar que la estimación que se realice de la $\mathrm{CRH}$ de la organización, con ítems pertenecientes a una mayor variedad de subconstructos, sea muy semejante a la obtenida con ítems de un menor número de ellos.

Hipótesis ${ }_{5}$. Se espera que el análisis de regresión simple realizado sobre los subíndices que componen los subconstructos de la $\mathrm{CRH}$ de la organización, permita identificar y diferenciar cuáles de ellos se relacionan de forma positiva o negativa, incluyendo en estos últimos a los subconstructos estrés y burnout.

Hipótesis 6. Cabe espera que los resultados obtenidos del análisis de regresión múltiple realizado sobre los subíndices que componen los subconstructos de la $\mathrm{CRH}$ de la organización, concuerden con los resultados obtenidos del análisis factorial realizado sobre los ítems que componen el índice de $\mathrm{CRH}$ de la organización.

\section{Metodología}

Para la valoración del término $\mathrm{CRH}$ se ha utilizado una muestra proveniente de una base de datos de 1.785 sujetos pertenecientes a 15 organizaciones diferentes del sector metalúrgico, químico y de comercio de todo el Estado español. Los análisis estadísticos realizados son:

- Análisis Correlacional ítems - subescalas de CRH de la organización.

- Análisis de fiabilidad de los ítems que componen la escala $\mathrm{CRH}$ mediante el alfa de Cronbach.

- Regresión simple aplicada sobre diferentes subconstructos que componen la $\mathrm{CRH}$, el coeficiente de determinación y expresión gráfica del scatter-plot resultante.

- Regresión múltiple mediante el método stepwise (paso a paso).

- Análisis Correlacional sobre distintas combinaciones de ítems que componen la $\mathrm{CRH}$ de la organización.

- Análisis factorial exploratorio (ML) realizados sobre submuestras de ítems que componen la $\mathrm{CRH}$ de la organización.

\section{Resultados}

La elaboración y construcción empírica del índice de Calidad de Recursos Humanos (CRH), se realizó siguiendo la lógica de la estructura del modelo Auditoría del Sistema Humano, desarrollado en la Universidad de Barcelona, España (Díaz de Quijano, 2000). De los 581 ítems que constituyen la base de datos global del $\mathrm{ASH}$, se realizó una primera selección de ellos, escogiendo a los que considerábamos que se encontraban asociados de mejor forma a una mayor o menor calidad general de los recursos humanos de la organización. En esta primera fase, que corresponde a la selección y evaluación subjetiva del investigador, se construyó un índice provisional, compuesto por el sumatorio de 150 ítems que considerábamos podían estimar adecuadamente la $\mathrm{CRH}$. Del mismo modo, se realizó una comprobación adicional. Distribuimos estos 150 ítems con el objetivo de construir tres subíndices de $\mathrm{CRH}$, cada uno de ellos independientes entre sí, pero con la salvedad de que se conservaran una cierta proporción en su distribución, que nos permitiera asegurar que pudieran ser comparables. El análisis correlacional realizado sobre ellos nos entregó valores elevados $(r<.90)$.

Posteriormente, realizamos una segunda comprobación. Establecimos pruebas de correlación entre estos tres índices calculados y el conjunto de los ítems del ASH - incluidos los que no habían sido utilizados inicialmente -, lo cual nos permitió depurar la composición definitiva del 
índice de $\mathrm{CRH}$. Debemos precisar que los criterios de decisión para establecer el índice final de $\mathrm{CRH}$ fueron tres, a saber:

- Que los ítems seleccionados tuvieran una alta correlación con el índice CRH. Con esta finalidad, establecimos un significativo punto de selección de los ítems que obtuvieron una más alta correlación $(r>.30, p<0.001)$,

- Una elevada capacidad discriminante del promedio del ítem entre los subgrupos de alta y baja $\mathrm{CRH}$, y

- Que los contenidos semánticos del ítem tuvieran una atribución clara de calidad.

La nueva escala de $\mathrm{CRH}$ quedó definitivamente compuesta por 175 ítems, los cuales fueron subdivididos con el objetivo de construir tres subíndices, cada uno de ellos independientes entre sí, pero con la salvedad de que se conservaran una cierta proporción en su distribución, de forma de asegurar que pudieran ser comparables. Se ha intentado mantener un equilibrio de todos ellos en las tres escalas de $\mathrm{CRH}$, lo cual en términos generales ha sido conseguido. Para la escala total de $\mathrm{CRH}$ se obtuvieron los siguientes valores:

\section{Figura 2}

\section{Estadísticos Descriptivos Escala Total de CRH}

Estadísticos
CUALIT
\begin{tabular}{|ll|r|}
\hline N & Válidos & 1292 \\
& Perdidos & 4 \\
Media & & 5,9406 \\
Mediana & & 6,0714 \\
Desv. típ. & & 1,3421 \\
Mínimo & & 1,83 \\
Máximo & & 9,82 \\
Percentiles & 25 & 5,0510 \\
& 50 & 6,0714 \\
& 75 & 6,8750 \\
\hline
\end{tabular}

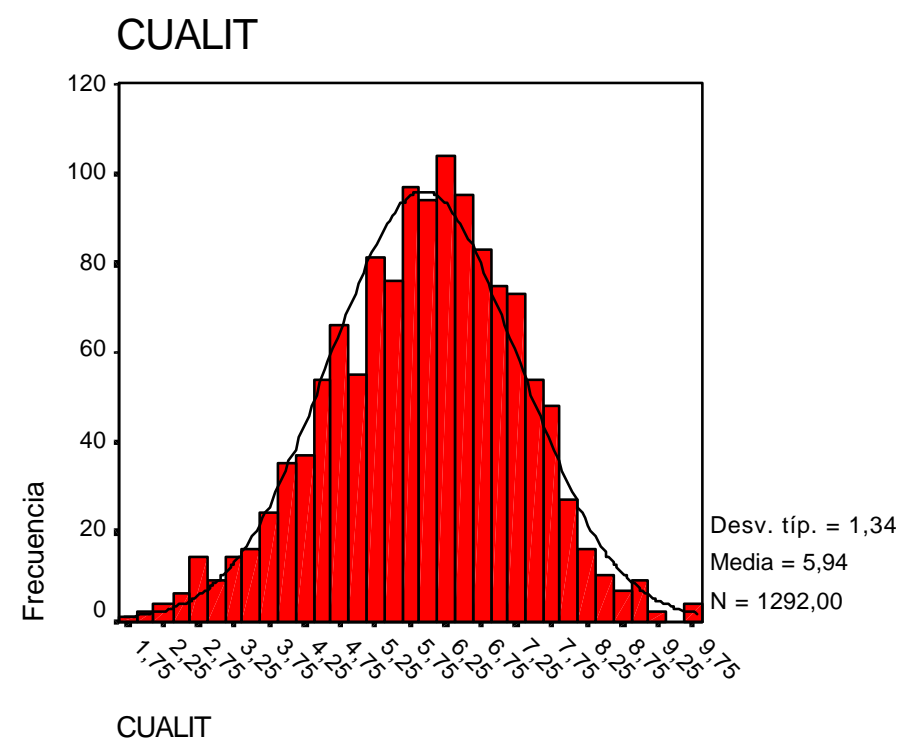

Una vez construidas las subescalas de $\mathrm{CRH}$, fueron sometidas a una nueva comprobación. Para tal efecto se realizaron estudios correlacionales entre cada una de ellas, los ítems y los índices del $\mathrm{ASH}$, así como comparaciones de medias, con el objetivo de corroborar la bondad del funcionamiento de la escala. Los resultados obtenidos nos entregaron una correlación significativa $(r>.35)$ entre los ítems y las escalas de $\mathrm{CRH}$; y así mismo, obtuvimos diferencias de medias significativas entre grupos extremos.

Posteriormente, realizamos un análisis de fiablidad de los ítems que componen la escala $\mathrm{CRH}$ mediante el alfa de Cronbach. Debemos admitir que el análisis se ha realizado de forma parcial. Ello se debe a que los ítems que componen la base de datos del ASH no siempre posee la 
homogeneidad estadística necesaria para realizar determinados análisis, puesto que se privilegia en cada estudio - intervención una selección de ítems que mejor se adaptan al contexto específico de las organizaciones. Ello conlleva, en algunas ocasiones, a una pérdida en la completitud de la base de datos global afectando de algún modo a las muestras de ítems y de sujetos sobre los que se realizan los análisis estadísticos. Por este motivo, nuestra opción fue realizar análisis de confiabilidad sobre los conjuntos de ítems que consideramos válidos.

\section{Tabla 1}

\section{Análisis parciales de fiabilidad (alfa de Cronbach)}

\begin{tabular}{|l|c|c|c|c|c|c|}
\cline { 2 - 7 } \multicolumn{1}{c|}{} & Análisis 1 & Análisis 2 & Análisis 3 & Análisis 4 & Análisis 5 & Análisis 6 \\
\hline № de ítems & 66 & 92 & 60 & 134 & 13 & 77 \\
\hline № de sujetos & 93 & 14 & 347 & 102 & 424 & 107 \\
\hline Alfa de Cronbach & .91 & .95 & .93 & .93 & .83 & .87 \\
\hline
\end{tabular}

Como se observa en la tabla nำ1, el análisis que incluye mayor número de ítems (134 de los 175 posibles -el 76,57\%-, sobre 102 sujetos) obtiene un alfa de .9346. Los restantes análisis parciales de fiabilidad arrojan alfas entre .8332 y .9524, que debe interpretarse como una buena confiabilidad de la escala. Del mismo modo, decidimos realizar análisis de confiabilidad para los índices de cada subconstructo que compone la $\mathrm{CRH}$. Debemos indicar que una vez realizado el análisis, encontramos puntuación alfa de .7892, valor que ciertamente no es tan bueno como el anterior, pero aún así sigue siendo válido.

A fin de conocer la dirección que adquiere la correlación entre los ítems e índices que componen la $\mathrm{CRH}$, realizamos una recategorización en cinco niveles de los índices del ASH, y posteriormente trasladamos los resultados de la expresión gráfica de los promedios en $\mathrm{CRH}$ por cada nivel del índice. A continuación presentamos algunos de los resultados obtenidos.

\section{Burnout}

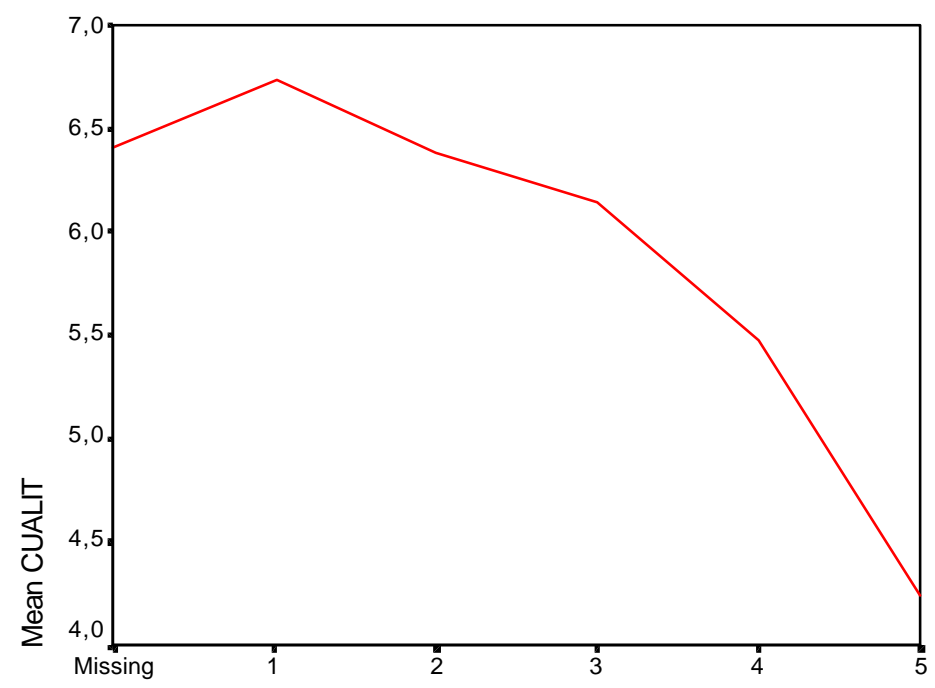

NTILES of BURNOUT 
Revista Mad. No.8. Mayo 2003. Departamento de Antropología. Universidad de Chile http://rehue.csociales.uchile.cl/publicaciones/mad/08/paper01.pdf

\section{Liderazgo Laissez-faire o No Liderazgo}

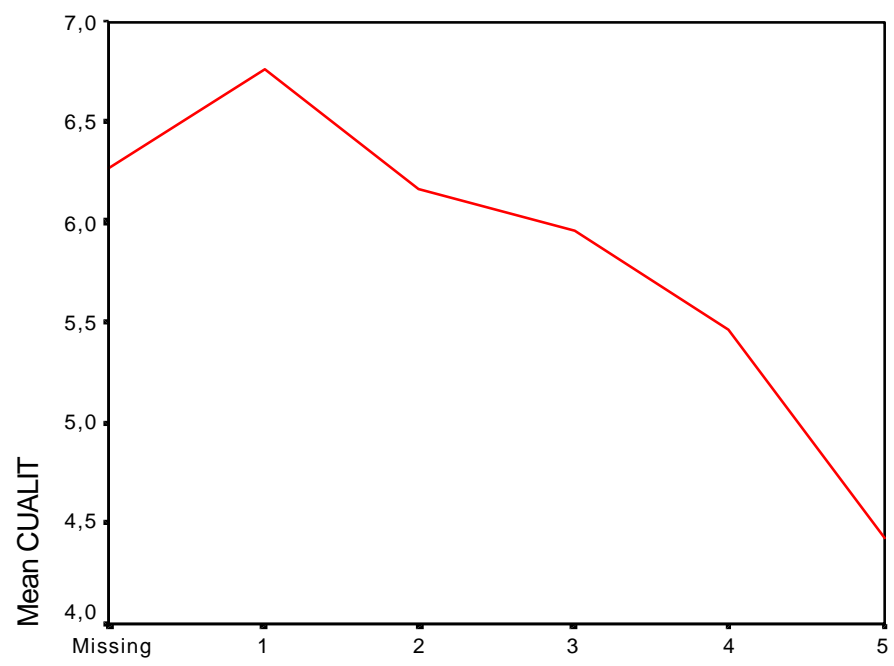

NTILES of LIDER3

Los resultados obtenidos indican que queda expresamente demostrada la relación negativa entre el índice de $\mathrm{CRH}$ y los índices correspondientes a los constructos estrés, burnout, liderazgo laissez-faire o no-liderazgo, cultura de orientación hacia el poder - evitación, cultura de orientación competitiva, conflicto de rol y sobrecarga de trabajo.

\section{Satisfacción}

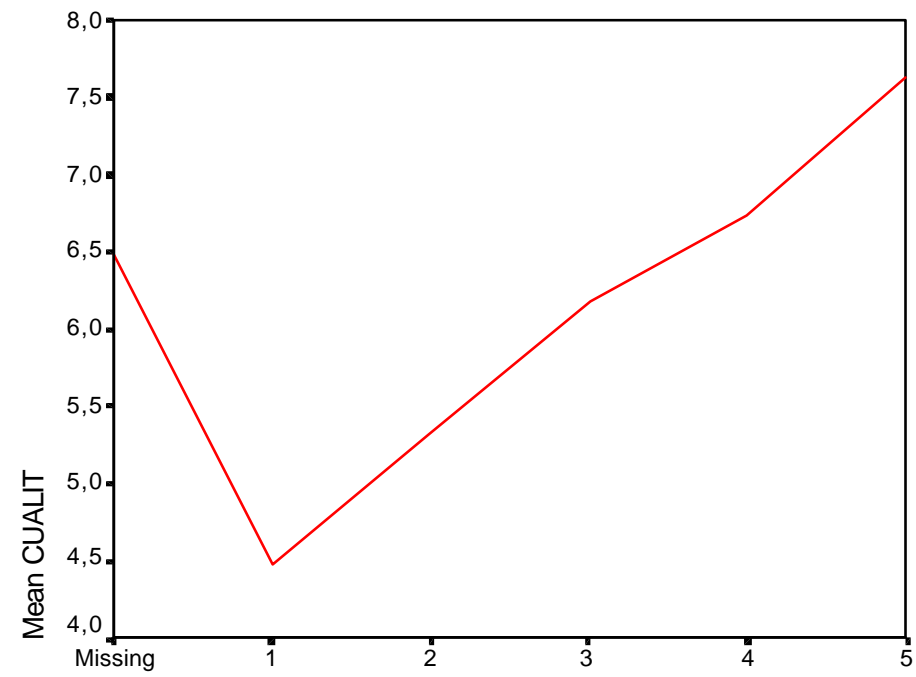

NTILES of SATISF 


\section{Liderazgo Tranformador}

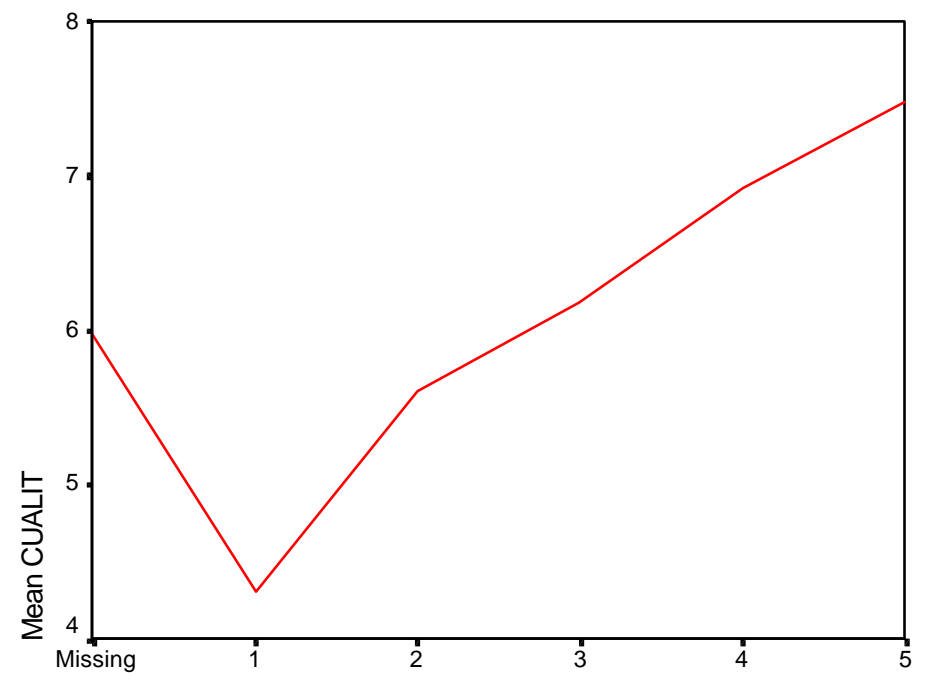

NTILES of LIDER1

Del mismo modo, los resultados obtenidos nos muestran la relación positiva entre el índice de $\mathrm{CRH}$ y los índices correspondientes a los constructos satisfacción, activación, compromiso, adecuación de la participación, liderazgo transformacional, motivación efectiva, potencial motivador del puesto, cultura orientada a la realización de las personas, autoeficacia, equidad, conciencia de resultados, significado percibido del puesto, claridad de rol, apoyo social, ocio y tiempo libre, cambio, grupalidad y cualidades del grupo para el rendimiento y la innovación.

Seguidamente, realizamos un análisis de regresión simple, haciendo participar el índice de CRH y los distintos subconstructos que lo componen. A continuación, presentamos algunos de los resultados del análisis de regresión simple realizado, los coeficientes de determinación para cada uno de ellos y la expresión gráfica del scatter-plot resultante.

\section{Liderazgo transformador}

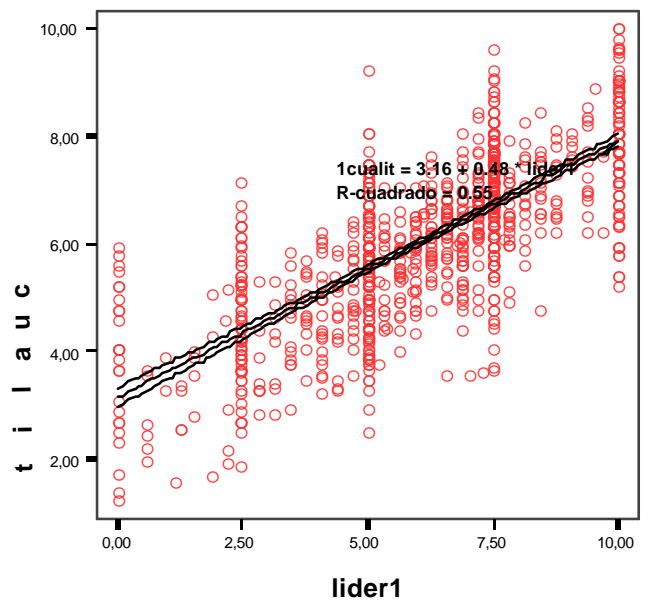

Regresión lineal con

Intervalo de predicción de la media al $95.00 \%$ 


\section{Satisfacción}

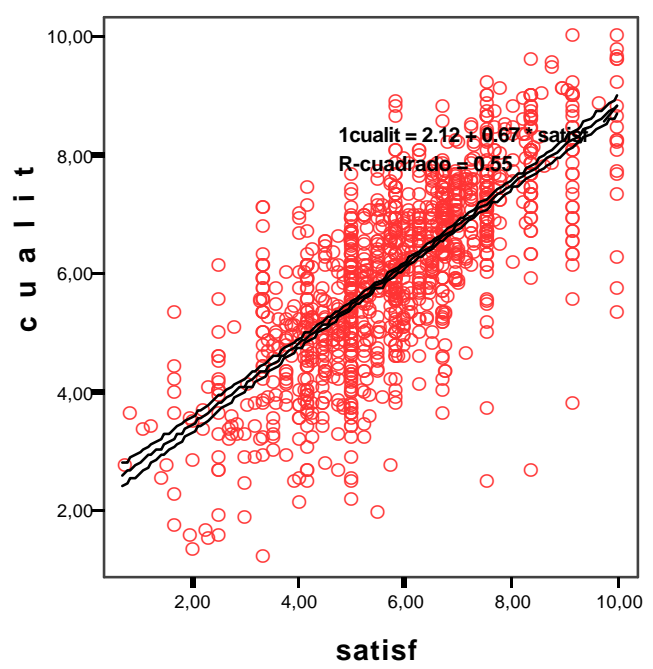

Regresión lineal con

Intervalo de predicción de la media al 95.00:

Los gráficos de scatterplot permiten observar con claridad el signo de la relación entre Calidad y los diversos índices del ASH así como la varianza explicada por cada índice sobre el constructo $\mathrm{CRH}$ a través de la puntuación R-Square. Los índices de mayor porcentaje ce varianza explicada son: Dirección Orientada a Relaciones $(\mathrm{R} 2=.61)$, Identificación y Compromiso $(\mathrm{R} 2=$ $.57)$, Liderazgo Transformador ( $R 2=.55)$, Satisfacción $(\mathrm{R} 2=.55)$, Compromiso $(\mathrm{R} 2=.52)$ y Cultura - Orientación Realización Personas $(\mathrm{R} 2=.50)$.

Posteriormente realizamos unos análisis de regresión múltiple por el método stepwise sobre la base de los seis índices que obtienen las $R 2$ más altos $(R 2>.5)$. La regresión múltiple de estos seis índices indica que, haciendo participar tan sólo al factor Liderazgo Transformador se consigue explicar un $65,4 \%$ de la varianza. Si se toman en consideración los seis índices, el modelo alcanza un $92 \%$. La secuencia que sigue dicho ajuste es la siguiente: Liderazgo transformador (65,4\%), Compromiso (19,7\%), Satisfacción $(2,6 \%)$, Cultura - Orientación Realización Personas (2,2\%), Dirección Orientada a Relaciones (1,4\%), Identificación y Compromiso $(0,7 \%)$. Estos resultados deben tomarse con cuidado y relativizar su importancia, puesto que la introducción de cada uno de los índices "paso a paso" (stepwise) produce diferencias significativas en el ajuste del modelo $(p<.000)$.

Como hemos indicado anteriormente, el índice $\mathrm{CRH}$ global opera sobre un total de 175 ítems, distribuidos entre las distintas dimensiones que conforman los constructos del ASH. A continuación, realizamos un diseño experimental de estimación de la $\mathrm{CRH}$ bajo 15 condiciones distintas repetidas en tres ocasiones. El objetivo de realizar este análisis, ha sido determinar si algunas combinaciones particulares de tems realizan mejores estimaciones de calidad que otras. A fin de mantener la equivalencia inicial de los grupos, es decir, asegurarnos que son similares entre sí al momento de realizar nuestras mediciones, describimos las condiciones de cálculo realizadas:

- 15 ítems aleatorios sobre el total de 175 ,

- 15 ítems, uno de cada subescala, buscando la máxima variabilidad inter - ítem, 
- Grupos de 10 ítems relativos todos ellos a distintos índices específicos (por ejemplo, Cambio, Liderazgo, Motivación, Satisfacción, Compromiso, Participación, Desarrollo Grupal, Burnout),

- Selección de ítems formulados de modo positivo frente a selección de ítems formulados de modo negativo en los cuestionarios,

- Selección de 15 ítems con mayor correlación inicial con el CRH frente a selección de 15 ítems con menor correlación.

\section{Tabla 2}

Correlaciones entre distintas condiciones de estimación de CRH (en conjuntos de 15 ítems sobre un total de 175) y la estimación global (Cualit)

\begin{tabular}{|l|c|c|c|c|c|c|}
\cline { 2 - 7 } \multicolumn{1}{c|}{} & $\begin{array}{c}15 \text { ítems } \\
1 \text { ítem de } \\
\text { cada } \\
\text { escala }\end{array}$ & $\begin{array}{c}15 \text { ítems } \\
\text { aleatorios }\end{array}$ & $\begin{array}{c}15 \text { ítems } \\
\text { positivos }\end{array}$ & $\begin{array}{c}15 \text { ítems } \\
\text { negativos }\end{array}$ & $\begin{array}{c}15 \text { ítems } \\
\text { de mayor } \\
\text { correlación } \\
\text { con CRH }\end{array}$ & $\begin{array}{c}15 \text { ítems } \\
\text { de menor } \\
\text { correlación } \\
\text { con CRH }\end{array}$ \\
\hline Prueba 1 & .83 & .78 & .66 & .72 & .68 & .53 \\
\hline Prueba 2 & .82 & .89 & .72 & .63 & .79 & .75 \\
\hline Prueba 3 & .79 & .78 & .79 & .47 & .70 & .61 \\
\hline \hline $\begin{array}{l}\text { PROMEDI } \\
\text { O }\end{array}$ & .81 & .82 & .72 & .61 & .72 & .63 \\
\hline
\end{tabular}

La correlación es significativa al nivel 0,01 (bilateral).

Los resultados que hemos obtenido son concluyentes; el rango de correlación entre los distintos estimadores y el de $\mathrm{CRH}$ oscilan entre .61 y .82, siendo el mejor estimador el obtenido por la combinación de 15 ítems aleatorios $(r=.82)$, seguido de la combinación que incluye un ítem de cada escala $(r=.81)$. Las combinaciones temáticas (ítems de una misma escala) oscilan entre .81 y .66. Los Ítems de Liderazgo y Cambio son los estimadores que mayor correlación alcanzan con el índice $\mathrm{CRH}(r=.81$ y $r=.80$, respectivamente). Los ítems de Burnout, así como la combinación de ítems de Compromiso nos entregan valores que oscilan entre .66 y .67, respectivamente.

Por último, realizamos dos análisis factoriales parciales sobre las sub-muestras de los ítems de $\mathrm{CRH}$. El propósito del análisis factorial es ayudar a los investigadores a descubrir e identificar las unidades o dimensiones, llamadas factores, que se encuentran detrás de cada medida. Los resultados del primer análisis factorial parcial se encuentran recogidos en la tabla $\mathrm{n}-3$. El análisis se realizó tomando en cuenta un total de 86 ítems, de los cuales se ilustran los primeros 36. La tabla nos muestra los tres primeros factores. El primer factor agrupa mayoritariamente ítems de identificación y compromiso organizacional; el segundo factor agrupa mayoritariamente ítems de estrés, activación y burnout; mientras que el tercer factor agrupa mayoritariamente ítems de satisfacción laboral y motivación. La secuencia que explica el porcentaje de la varianza explicada es la siguiente: primer factor $(34 \%)$, segundo factor $(6,6 \%)$ y tercer factor $(5,1 \%)$. Los tres primeros factores explican un $45,73 \%$ de la varianza. 
KMO and Bartlett's Test

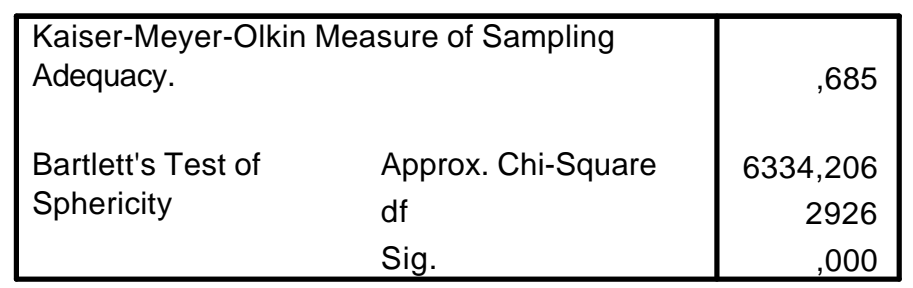

\section{Tabla 3}

\section{Ejemplos del Análisis Factorial}

\begin{tabular}{|c|c|c|c|c|c|}
\hline ITEM & VAR & Commun & \begin{tabular}{|l} 
FACTOR \\
1
\end{tabular} & \begin{tabular}{|l} 
FACTOR \\
2
\end{tabular} & $\begin{array}{l}\text { FECTOR } \\
3\end{array}$ \\
\hline $\begin{array}{l}\text { Cuando alguien critíca mi } \\
\text { organización lo siento como } \\
\text { un insulto personal }\end{array}$ & IC5 & .838 & ,808 & $\begin{array}{l}8,885 \mathrm{E}- \\
02\end{array}$ & ,180 \\
\hline $\begin{array}{l}\text { Cuando alguien alaba a esta } \\
\text { organización lo siento como } \\
\text { un cumplido personal }\end{array}$ & IC3 & .788 & ,783 & 117 &, 120 \\
\hline $\begin{array}{l}\text { Me siento orgullosos cuando } \\
\text { les digo a otros que soy } \\
\text { miembro de mi organización }\end{array}$ & IC37 & .834 & ,741 & 234 & ,181 \\
\hline Molesto-Contento & ST6 & .833 & ,266 &, 775 & ,263 \\
\hline Abatido-Animado & AC9 & .783 & ,107 & ,773 &, 111 \\
\hline Temeroso-Seguro & ST12 & .834 & $\begin{array}{l}8,513 \mathrm{E}- \\
02\end{array}$ & ,711 & $\begin{array}{l}-4,835 \mathrm{E}- \\
02\end{array}$ \\
\hline $\begin{array}{l}\text { Los elogios que recibe } \\
\text { cuando obtiene buenos } \\
\text { resultados }\end{array}$ & S7 & .837 & ,213 & 128 & ,799 \\
\hline Será felicitado por su jefe & M35 & .720 & ,175 & ,119 & 762 \\
\hline $\begin{array}{l}\text { El apoyo que } \\
\text { parte de sus } \\
\text { inmediatos }\end{array}$ & S4 & .847 & ,144 &, 113 &, 753 \\
\hline
\end{tabular}

Los resultados del segundo análisis factorial parcial también son concordantes. El análisis se realizó tomando en cuenta un total de 66 ítems, de los cuales se ilustran los primeros 38. La tabla nos muestra los tres primeros factores. El primer factor agrupa mayoritariamente ítems de identificación y compromiso organizacional; el segundo factor agrupa mayoritariamente ítems de estrés, activación y burnout; mientras que el tercer factor agrupa mayoritariamente ítems de satisfacción laboral y motivación. La secuencia que explica el porcentaje de la varianza es la siguiente: primer factor $(27,6 \%)$, segundo factor $(12,1 \%)$ y tercer factor $(8,7 \%)$. En total, los tres primeros factores explican un $48,43 \%$ de la varianza.

Aunque reconocemos que estos análisis factoriales son parciales, los resultados que nos entregan se encuentran en concordancia con los resultados encontrados en la regresión múltiple realizada con los índices que explicaban un mayor porcentaje de la varianza, ello nos 
Revista Mad. No.8. Mayo 2003. Departamento de Antropología. Universidad de Chile http://rehue.csociales.uchile.cl/publicaciones/mad/08/paper01.pdf

permite realizar una primera aproximación a los factores que conforman la $\mathrm{CRH}$ de la organización.

\section{Comprobación de las hipótesis}

Del análisis de los resultados obtenidos en las distintas pruebas realizadas podemos realizar la comprobación de las hipótesis nuestro estudio.

Hipótesis ${ }_{1}$. Efectivamente, el análisis de fiabilidad de los ítems que componen la escala $\mathrm{CRH}$, realizado mediante el alfa de Cronbach, dieron como resultado una alta confiabilidad de la escala. El análisis realizado sobre el mayor número de ítem (134 de los 175 posibles) obtuvo un alfa de .9346. Otros análisis parciales de fiabilidad también registraron alfas que resultan ser adecuados y que oscilan entre .8332 y .9524 .

Hipótesis 2. Los análisis realizados también comprueban esta hipótesis, al obtenerse una puntuación alfa de .7892 que, siendo más baja que la anterior, debe ser entendida como adecuada.

Hipótesis ${ }_{3}$. Efectivamente, se comprueba la hipótesis que la estimación que se realice de la $\mathrm{CRH}$ de la organización con un número menor de ítems, ha de ser muy semejante a la obtenida con un número mayor de ellos. Los resultados obtenidos nos indican que el rango de correlación entre los distintos estimadores realizados y el CRH oscilan entre .61 y .82, siendo el mejor estimador el obtenido por la combinación de 15 ítems aleatorios $(r=.82)$. Estimadores compuestos por combinaciones de 10 ítems también muestras correlaciones elevadas que oscilan entre .66 y .81, siendo el mejor estimador la combinación que incluye tan sólo ítems de liderazgo $(r=.81)$.

Hipótesis 4. Efectivamente, se comprueba la hipótesis que la estimación que se realice de la $\mathrm{CRH}$ de la organización con ítems pertenecientes a una mayor variedad de subconstructos, ha de ser muy semejante a la obtenida con ítems de un menor número de ellos. Los resultados obtenidos nos indican que el rango de correlación entre los distintos estimadores realizados con un ítem de cada subconstructo o de forma aleatoria $(r=.81$ y $r=.82$, respectivamente) resultan ser tan elevados como las combinaciones de ítems de tan solo un constructo, como es el caso del liderazgo y el cambio ( $r=.81$ y $r=.80$, respectivamente).

Hipótesis ${ }_{5}$. El análisis de regresión simple realizado sobre los subíndices que componen los subconstructos de la $\mathrm{CRH}$ de la organización, nos ha permitido comprobar la dirección negativa de la correlación de los ítems e índices de estrés y burnout con el de $\mathrm{CRH}$, los que explican un $33 \%$ y un $39 \%$ de la varianza respectivamente. Se encontró una relación negativa entre el índice de $\mathrm{CRH}$ y los índices correspondientes a los constructos cultura de orientación hacia el poder - evitación, cultura de orientación competitiva, conflicto de rol, liderazgo laissez-faire o no liderazgo y sobrecarga de trabajo.

Del mismo modo, quedó demostrada la relación positiva entre el índice de $\mathrm{CRH}$ y los índices correspondientes a los constructos satisfacción, activación, compromiso, adecuación de la participación, liderazgo transformacional, motivación efectiva, potencial motivador del puesto, cultura orientada a la realización de las personas, autoeficacia, equidad, conciencia de resultados, significado percibido del puesto, claridad de rol, apoyo social, ocio y tiempo libre, cambio, grupalidad y cualidades del grupo para el rendimiento y la innovación. 
Hipótesis ${ }_{6}$. Se confirma la hipótesis, aunque de forma parcial. Los resultados obtenidos del análisis de regresión múltiple se han realizado con los índices de los subconstructos Dirección Orientada a las Relaciones, Identificación y Compromiso, Liderazgo Transformador, Satisfacción, Compromiso y Cultura de orientación a la realización de las personas. La regresión realizada sobre estos seis índices indica que, haciendo participar el factor Liderazgo Transformador se consigue explicar un $65,4 \%$ de la varianza, y si se toman en conjunto los seis índices, el modelo alcanza un $92 \%$.

Los resultados que entregan los análisis factoriales parciales realizados sobre conjuntos de ítems (86 ítems en el primer factorial y 66 ítems en el segundo), nos muestran que los tres primeros factores explican un $45,73 \%$ y un $48,43 \%$ del total de la varianza, respectivamente. El primer factor agrupa mayoritariamente ítems de identificación y compromiso organizacional; el segundo factor agrupa mayoritariamente ítems de estrés, activación y burnout; mientras que el tercer factor agrupa mayoritariamente ítems de satisfacción laboral y motivación. Como puede apreciarse, existen varias similitudes entre los resultados que presentan ambos análisis, si se tiene en consideración que en el análisis factorial no se hicieron participar ítems de liderazgo. Sin embargo, en ningún caso pueden ser considerados como concluyentes y deben relativizarse a su respectivo contexto, por ello decimos que la hipótesis de confirma de forma parcial.

\section{Conclusiones}

A fin de realizar una adecuada aproximación al término $\mathrm{CRH}$ de la organización, realizamos una serie de pruebas estadísticas a partir de la construcción de una escala de $\mathrm{CRH}$. Nos complace decir que los distintos resultados expuestos son congruentes con las hipótesis teóricas y metodológicas enunciadas.

De los resultados obtenidos se destaca que es posible elaborar una escala de $\mathrm{CRH}$ que posea una elevada confiabilidad, medida mediante el análisis del alfa de Cronbach, sin importar si la escala se construye con ítems de los subconstructos que la componen o con sus índices. Del mismo modo, hemos podido constatar que cada uno de los subconstructos que componen la $\mathrm{CRH}$ puede ser - a un mismo nivel - un estimador adecuado. El análisis de regresión simple nos ha permitido identificar y diferenciar cuáles son los subíndices que se relacionan de forma positiva y negativa con la $\mathrm{CRH}$. El análisis de regresión múltiple y análisis factorial nos permitió dilucidar los principales subconstructos implicados en la $\mathrm{CRH}$ de la organización.

Nuestro estudio, por cierto, no nos entrega conclusiones definitivas. Las limitaciones propias a toda investigación no nos permiten, hasta el momento, dilucidar de forma tan clara como quisiéramos la estructura interna del índice $\mathrm{CRH}$. En este sentido, la $\mathrm{CRH}$ tal como aquí se ha tratado debe interpretarse más bien como un índice holístico y globalizador, cuya finalidad y funcionalidad permite sintetizar la multiplicidad de datos implicados en el modelo ASH.

Somos conscientes de nuestras limitaciones y reconocemos que se vuelve necesario realizar mayores esfuerzos científicos y analíticos a fin de dilucidar, con un nivel mayor de certeza, los factores constituyentes de la $\mathrm{CRH}$ de la organización. Sin embargo, consideramos que la $\mathrm{CRH}$ tiene una gran importancia teórica y práctica. Hoy en día se vuelve necesario disponer de constructos e índices que sean capaces de aglutinar y acotar de forma sintética conceptos y variables propios de nuestras disciplinas. Al mismo tiempo, esos constructos e índices deben permitirnos intervenir adecuadamente en las organizaciones, por medio de auditorías completas, con un ahorro considerable de tiempo en la recogida de la información y de dinero para las organizaciones. 


\section{Bibliografía}

BATEMAN, T. S. Y STRASER, S. (1984). A Longitudinal analysis of the antecedents of organizational commitment. Academy of Managenment Journal, 27 (1), 95-112.

DÍAZ DE QUIJANO, S. (2000). Organizaciones: ¿qué son?, ¿cómo han sido pensadas?, su comportamiento, su evaluación. Barcelona: Bardenas.

FARKAS, A. J. Y TETRICK, L. E. (1989). A three-wave longitudinal analysis on the causal ordering of satisfaction and commitment on turnover decision. Journal of Applied Psychology, 74, páginas 855-868.

FLORES-GUERRERO, R. (2003). La Calidad de los Recursos Humanos de la Organización: dimensiones, conceptos, medidas y relaciones. Tesis para obtener el grado de Doctor en Psicología, Universidad de Barcelona, Barcelona.

\section{Notas}

(1) Este es el caso, por ejemplo, del modelo europeo de evaluación de la excelencia organizacional (EFQM) y del modelo norteamericano (Malcom Baldrige). 